Ann. Génét. Sél, anim., I969 1 (2), I3I-r33.

\title{
ÉTUDE D'UN GÈNE DE NANISME LIÉ AU SEXE CHEZ LA POULE
}

\author{
II. - FonCtionNEMENT THYROÏDIEN \\ P. MÉRAT et J. GUILLAUME \\ avec la collaboration technique de G. Coquerelle \\ Station centrale de Génétique animale, \\ Centre national de Recherches zootechniques, 78-Jouy-en-Josas \\ Station de Recherches avicoles, \\ Centre de Recherches vétérinaires et zootechniques \\ Domaine de l'Orfrasière, 37-Nouzilly \\ Institut national de la Recherche agronomique
}

\section{SOMMAIRE}

L'hypothyroïdisme de poules porteuses d'un gène de nanisme lié au sexe est suggéré.

D'après VAN TIENhoven et al. (I966), l'observation histologique suggère une moindre activité thyroïdienne liée, chez la poule, à la présence du gène $\mathrm{dw}$, récessif lié au sexe réduisant la taille.

Les observations suivantes sur notre troupeau de Jouy paraissent concorder avec la suggestion de ces auteurs :

a) Le poids des thyroïdes, en pour cent du poids corporel, est réduit en présence de dw : sur ro couples de " nains " et de " normaux " âgés de I2 semaines, en $\mathrm{I} 966$, ce pourcentage était de $3,7 \cdot \mathrm{IO}^{-4}$ pour les premiers, de $5, \mathrm{I} \cdot \mathrm{Io}^{-4}$ pour les seconds $(\mathrm{P}<0,05)$.

b) Le pourcentage de graisse abdominale, sur les mêmes animaux, est, pour les nains, de plus du double des normaux $(3,2 \%$ contre $\mathrm{r}, 3 \%, \mathrm{P}<0,0 \mathrm{r})$.

Par comparaison, au même âge, le poids relatif du foie et des surrénales ne diffère pas notablement suivant le génotype. 
c) En novembre 1967 , nous avons évalué le taux de sécrétion thyroïdienne sur 40 qo $\mathrm{dw}$ - et 40 q $\mathrm{Dw}$ - (apparentées aux précédentes) mises en cages individuelles avec quatorze heures de lumière à leur entrée en ponte en octobre. La méthode utilisée, décrite par MELLEN et WENTWORTH (I960), consiste à répartir les animaux en deux groupes " témoins " (Aliment normal - Aliment à I \% de thiouracile) et trois lots recevant respectivement, outre le deuxième aliment, une injection journalière de $0,5 \gamma, I, 5 \gamma$ et $2,5 \gamma$ de thyroxine par roo grammes de poids vif. Pour chaque génotype, la droite de régression du poids des thyroïdes en fin d'expérience sur la dose reçue servait à évaluer le taux normal de thyroxine excrétée. Les sécrétions estimées sont $2,59 \gamma$ de thyroxine par roo grammes de poids vif pour les "normales" et une valeur proche de zéro pour les naines.

Quoique la précision des estimations soit assez faible, du fait de la taille de l'échantillon, et que les valeurs absolues données par la méthode prêtent à certaines critiques, dans le sens d'une sous-estimation (MELLEN et WENTwORTH, I960), le résultat de la comparaison entre les deux génotypes est assez clair. Il existe une différence du taux de sécrétion hautement significative en faveur des normales.

d) Utilisation alimentaire et métabolisme comparés de poussins normaux et nains (GUILLAUME, rg69, à publier).

Nous avons comparé des poulettes " normales " ou " naines " issues d'un croisement pedigree expérimental. Le gain de poids, la consommation et divers critères de l'utilisation de l'aliment global, des protéines et de l'énergie ont été enregistrés. Un seul aliment, de type usuel, modifié à cinq semaines, a été distribué au cours de l'expérience qui a duré neuf semaines.

Comparées à des poulettes normales de même poids, les poulettes " naines " consomment autant d'aliment et croissent bien entendu moins vite. L'indice de consommation s'en trouve détérioré ainsi que le coefficient d'utilisation protidique. L'énergie métabolisable des régimes n'est pas affectée. Le besoin d'entretien, le métabolisme au repos et vraisemblablement le métabolisme basal, sont réduits par le gène $d w$. L'“ indice de consommation de croissance " est détérioré plus encore que l'indice de consommation global, mais ce phénomène provient en fait essentiellement d'une augmentation de la teneur en lipides des tissus synthétisés. L'utilisation énergétique mesurée par le rapport énergie retenue/énergie métabolisable ingérée est en faveur des poulettes " naines ". Les modifications en sens inverse des coefficients d'utilisation de l'énergie et des protéines seront discutées ultérieurement en relation avec la nature relativement boulimique des individus dw.

On constate que les " naines " ont une consommation d'oxygène beaucoup plus régulière dans le temps, ce qui est l'indice d'un naturel plus calme, confirmant d'ailleurs une impression subjective. Plusieurs des faits observés sont également en faveur d'un hypothyroïdisme des sujets dw.

e) Autres observations :

La durée d'incubation ne paraît sensiblement différente (du moins de manière suffisante pour être décelable) ni entre embryons normaux et nains, ni entre mères Dw- et $\mathrm{dw}$-, d'après nos propres résultats et ceux de Cochez au Magneraud (communication personnelle). 
Des dissections faites à 1'éclosion (DELPECH, I968) montrent un poids du poussin entier légèrement mais non significativement plus grand pour le génotype normal $(36,5$ contre $35,4 \mathrm{~g})$. Par contre, le vitellus pèse en moyenne $3,6 \mathrm{~g}$ pour les seconds contre $2,6 \mathrm{~g}$ pour les premiers. La résorption du jaune paraît donc se faire moins vite pour le poussin $\mathrm{dw}$. Ceci est confirmé par une deuxième série d'observations, du moins lorsque la mère est $\mathrm{Dw}$ - et, par là, apporte un vitellus de taille normale.

Une observation fortuite (poules ayant eu leur plumage mouillé) suggérait que les poules $d w$ - mettaient plus longtemps à rétablir leur température normale, alors, que les Dw-. Il semble par ailleurs que, dans les conditions ordinaires, aucune différence de sensibilité au froid ne se manifeste. Nous 1'avons vérifié sur des poussins normaux et nains élevés à une température inférieure à la normale.

Reçu pour publication en mai 1969.

\section{SUMMARY}

STUDIES ON A SEX-LINKED * DWARF * GENE IN THE FOWL

\section{II. - THYRoID ACTIVITY}

Hypothyroidism in sex-linked dwarf fowls is suggested by a reduced thyroid size in per cent, a much higher fat percentage, a lower secretion as estimated by the goiter-prevention method, and finally a lower metabolic rate among young chicks.

Incubation time does not seem to be appreciably modified, but resorption of the yolk is slower for dwarf chicks.

\section{RÉFÉRENCES BIBLIOGRAPHIQUES}

Cocrez L. P. Communication personnelle.

DELPECH P. Communication personnelle.

GUILLAUME J., 1969. Utilisation alimentaire et métabolisme comparés de poussins normaux et nains (dw). Ann. Biol. anim. Bioch. Biophys. (à publier).

MeLlen W. J., Wentworth B. C., I96o. Comparison of methods for estimating thyroid secretion rate in chickens. Poult. Sci., 39, 678-686.

Van Tienhoven A., Wimliamson J. H., Tomlinson M. C., Macinnes K. L., rg66. Possible role of the thyroid and the pituitary glands in sex-linked dwarfism in the fowl. Endocrinology, 78, 950-957. 